Tropical Journal of Pharmaceutical Research April 2016; 15 (4): 833-839

ISSN: $1596-5996$ (print); 1596-9827 (electronic)

(C) Pharmacotherapy Group, Faculty of Pharmacy, University of Benin, Benin City, 300001 Nigeria.

All rights reserved.

Available online at http://www.tjpr.org

Original Research Article

http://dx.doi.org/10.4314/tjpr.v15i4.24

\title{
Analysis of only 0-1 min clip or 1-4 min Clip for focal liver lesions during contrast-enhanced ultrasonography
}

\author{
Wei Zhang ${ }^{1}$, Hui-Ming $\mathrm{Yi}^{1}, \mathrm{Xi} \mathrm{Ai}^{2}$, Kai-Yan $\mathrm{Li}^{1}$ and You-Bin Deng ${ }^{1 *}$ \\ ${ }^{1}$ Department of Medical Ultrasound, ${ }^{2}$ Hepatic Surgery Center, Tongji Hospital, Tongji Medical College, Huazhong University of \\ Science and Technology, 1095 Jiefang Road, Wuhan 430030, PR China \\ *For correspondence: Email: docdengyb@163.com; Tel: +86-27-83663554; Fax: +86-27-83662693
}

\begin{abstract}
Purpose: To evaluate the reliability of analysis of only $0-1 \mathrm{~min}$ clips and 1-4min clips versus the entire clips in performing contrast-enhanced ultrasonography (CEUS) of focal liver lesions (FLLS).

Methods: Contrast-enhanced ultrasonography (CEUS) examinations of 43 single FLLs were performed. All clips were analyzed in three ways, the entire clips, 0-1 min clips and 1-4 min clips, benign or malignant diagnosis and pathological diagnosis of each FLL were concluded by the three ways subsequently.

Results: The results of correct diagnosis were assessed using Chi-square test. There was no difference with regard to benign or malignant diagnosis, between $0-1$ min clips and the entire clips, or between 1-4 min clips and the entire clips ( $p=0.243$ and $p=0.747$, respectively). Moreover, no significant differences in pathological diagnosis existed between 0-1min clips and the entire clips, and 14 min clips versus entire clips $(p=0.808$ and $p=0.808$, respectively). No significant differences existed among CEUS entire clip, 0-1min clip and 1-4min clip in identifying FLLs, and based on which the diagnosis of two different FLLs during CEUS with only one injection of contrast agent can be available. Conclusion: Only 0-1min clips or 1-4 min clips can be used to instead of the entre clip in performing CEUS of FLLS.
\end{abstract}

Keywords: Focal liver lesions, Contrast-enhanced ultrasonography, Ultrasonic diagnosis, Clips

Tropical Journal of Pharmaceutical Research is indexed by Science Citation Index (SciSearch), Scopus, International Pharmaceutical Abstract, Chemical Abstracts, Embase, Index Copernicus, EBSCO, African Index Medicus, JournalSeek, Journal Citation Reports/Science Edition, Directory of Open Access Journals (DOAJ), African Journal Online, Bioline International, Open-J-Gate and Pharmacy Abstracts

\section{INTRODUCTION}

Contrast-enhanced ultrasonography (CEUS) is a widespread imaging procedure for investigating the liver parenchyma. The injection of ultrasound contrast agents (USCA) and the use of specialized imaging techniques now allow to display tissue macro and micro-vascularization similarly to what is seen with contrast- enhanced computed tomography (CECT) or magnetic resonance imaging (CEMRI) [1]. Ultrasound contrast agents present a pure intravascular distribution and do not leak in the interstitial space but persist in the sinusoids and portal vessels without the evidence of any equilibrium phase [2]. The peculiar hepatic vascularization allows defining different vascular phases. The arterial phase starts from $10-20 \mathrm{~s}$ after the injection of MBs, lasts for approximately $10-15 \mathrm{~s}$ and provides information on the degree and pattern of vascularity. The portal phase then lasts for 2 min after the start of injection, whereas the subsequent late phase lasts for up to 4-6 min after injection, until microbubble clearance from the liver parenchyma. Both portal phase and late phase provide information about the wash-out of focal liver lesions (FLLs) compared to the surrounding parenchyma (the term wash-out 
describes a transition from some enhancement of a lesion, either iso- or hyper enhancement, to a clear hypoenhancement) [3].

During the last few years, many studies have investigated the CEUS patterns of FLLs, establishing their typical behavior in the arterial, portal and venous phases [3]. Several papers have described the general capabilities of CEUS in improving focal liver lesion characterization [2]. CEUS is now a reliable imaging tool with wellestablished applications in the characterization of liver lesions with the possibility of prolonged liver ultrasound examination, and is easily performed.

One limitation of CEUS in comparison to multiphase CT and MR imaging is the fact that only one liver lesion can be examined at a time as the transducer has to be kept still during the examination, and further contrast injections are necessary to characterize additional liver tumors. To date, a single clip over $180 \mathrm{~s}$ is required to analyze all phases (arterial, venous and late phases) of contrast behavior. The process lasts a long time and thus has to be performed off-line in "back-office" work and the analysis of entire video clips is very time-consuming and demanding in terms of technical resources, thus limiting potential applications in routine clinical use especially in assessing two different FLLs. In the attempt to simplify the process and to reduces acquisition time associated with CEUS, the entire clips of CEUS were divided into two clips, $0-1 \mathrm{~min}$ clips and $1-4 \mathrm{~min}$ clips, then the ratio of correct diagnosis and the statistical differences were analyzed in this study to evaluate the reliability of analysis of only $0-1 \mathrm{~min}$ clips and 1 $4 \mathrm{~min}$ clips versus the entire clips.

\section{EXPERIMENTAL}

\section{Patients and lesions}

All patients with single FLL who had undergone pathological examination originating from operation or biopsy and previously assessed with CEUS were considered eligible for this study. Patients with any of the following conditions were excluded:

1. Previous adverse reactions to Sonovue (Bracco Imaging, Milan, Italy),

2. Severe pulmonary hypertension;

3. Pregnancy or breastfeeding conditions;

4. Portal vein thrombus and postcava thrombus;

5. Evidence of critical illness or medical instability;
6. History of operation and minimally invasive therapy;

7. Poor image quality of the FLL.

Written informed consent was obtained from all patients before the examination, and the study was approved by the Institutional Review Board and Ethics Committee of Tongji Hospital, Tongji Medical College, Huazhong University of Science and Technology (no. TJ-C20121213). All CEUS studies were performed for clinical purposes and were not performed merely for the purpose of the current study.

\section{Imaging protocol}

Vivid E9 (GE, America) equipped with a $3.5 \mathrm{MHz}$ harmonic-imaging transducer was used for CEUS and clip recording. Initially, a B-mode scan was performed to identify the best approach to the lesion. This consisted in choosing a scan in which the lesion was located in the mid-near field and remained visible during respiratory movements. The choice of the mid-near field was made based on previous findings, showing that this region provides the highest reproducibility. Thereafter, a bolus of $2.4 \mathrm{ml}$ of Sonovue (Bracco Imaging, Milan, Italy), a second generation contrast agent composed of microbubbles of sulphur hexafluoride, was injected intravenously in an antecubital vein with a 22-gauge angiocatheter, extension tubing and a three-way stopcock., followed by $5 \mathrm{ml}$ flush of $\mathrm{NaCl}(0.9 \%)$ in bolus. Zero time was recorded at the completion of Sonovue flush. A continuous video clip of CEUS was acquired (duration 3-4min) following contrast injection. All investigations were performed in the same standardized way by the same expert operator, with 10 years of experience in CEUS.

\section{Imaging analysis}

The CEUS clips were analyzed in 3 ways: (a) the entire clips, (b) 0-1min clips, referred to here as artery and earlier portal phase methodology, (c) 1-4min clips, referred to here as later portal phase and late phase methodology. The CEUS clips were divided by an external operator, following a training period.

All images, including grayscale, color Doppler ultrasound images and CEUS clips were evaluated in DICOM format with computer workstation. Two blinded radiologist readers, both with 5 years of experience in CEUS, independently reviewed all the ultrasound examinations. Both readers were blinded to clinical features, laboratory examinations and other imaging findings. Then readers were asked 
to provide the most likely diagnosis (i.e., benign or malignant diagnosis, pathological diagnosis or indeterminate) for each focal liver lesion according to standard diagnostic criteria $[3,4]$.

\section{Statistical analysis}

Collected data were entered into a common spreadsheet and summary statistics were calculated. Categorical variables were assessed using the chi-square test. All the data were analyzed with SPSS 18.0. The results are expressed as mean \pm standard deviation (SD) for each measurement. $P<0.05$ was considered statistically significant.

\section{RESULTS}

Contrast Tuned Imaging software was utilized for contrast detection (Figure 1); dynamic range set at 7 , mechanical index at 0.03 , acoustic power at $40 \mathrm{kPa}$, frame rate at $15 \mathrm{~Hz}$, sectorial gains at zero. Visualization was always kept in dual mode display.

In our present investigation, 43 patients including 29 men and 14 women, who ranged in age from 21 to 78 years, with satisfactory image quality were analyzed. The differences in the clinical characteristics are reported in Table 1. The study comprised 43 lesions (16 hepatocellular carcinomas, 2 intrahepatic cholangiocarcinomas, 2 regenerative nodules, 7 liver metastases carcinomas, 4 focal nodular hyperplasias, 6hemangiomas, 1 hepatocellular adenoma, 3 inflammatory pseudotumors, 1 liver abscess, 1 hematoma). The final diagnosis was established using operation and biopsy. The median diameter of the lesions was $4.2 \mathrm{~cm}(0.8-14.4$ $\mathrm{cm})$.

The results of the entire clips, 0 -1min clips and 1$4 \mathrm{~min}$ clips concerning benign and malignant diagnosis as well as pathological diagnosis are shown in Tables 2 and 3 . There were no differences with regard to benign or malignant diagnosis between $0-1 \mathrm{~min}$ clips versus the entire clips as well as 1-4min clips versus the entire clips ( $p=0.243$ and $p=0.747$, respectively) (Table 4). Moreover, no significant differences in pathological diagnosis existed between 0-1min clips versus the entire clips and $1-4 \mathrm{~min}$ clips versus the entire clips $(p=0.808$ and $p=0.808$, respectively) (Table 4 ).

\section{DISCUSSION}

So far, many studies have investigated the CEUS patterns for FLLs examination, establishing their typical behavior in the arterial, portal and venous phases [3]. Malignancies typically show low echo-signal intensity at late phase [5,6], and the contrast pattern that characterizes benign FLLs is the absence of wash-out in the portal-venous and late phases [2]. Besides, benign and malignant FLLs may occur in cirrhosis and fatty liver $[7,8]$.

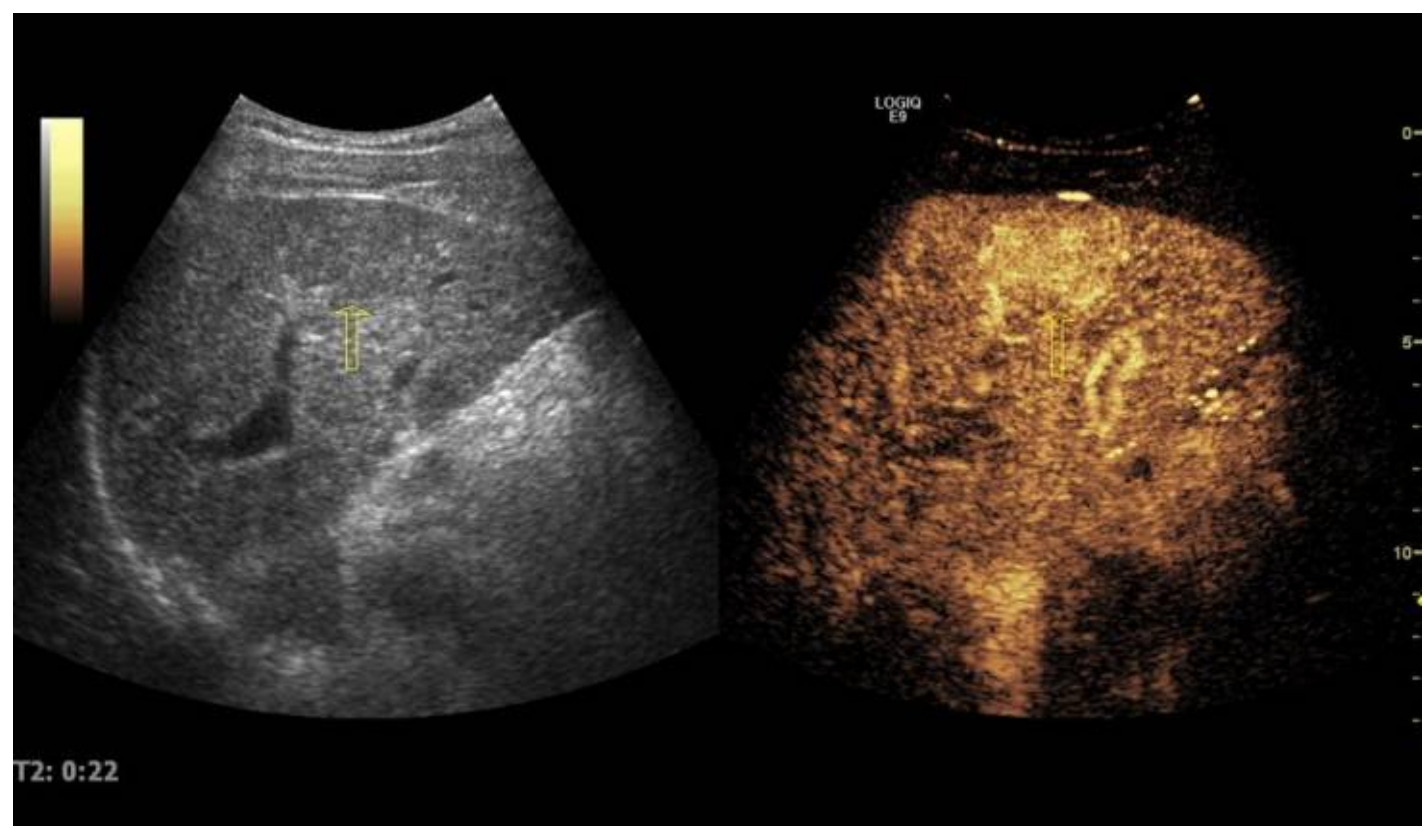

Figure 1: Contrast tuned imaging. On the left of the screen, the hypoechoic focal liver lesion-hepatocellular carcinoma (arrow) is displayed in B-mode modality, while on the right the lesion (arrow) is displayed in Contrast Tuned Imaging ( $\mathrm{CnTI})$ technology. Thus the Real-time CEUS and the gray-scale image can be performed at the same time 
Table 1: Focal liver lesions (FLLs) used in this study

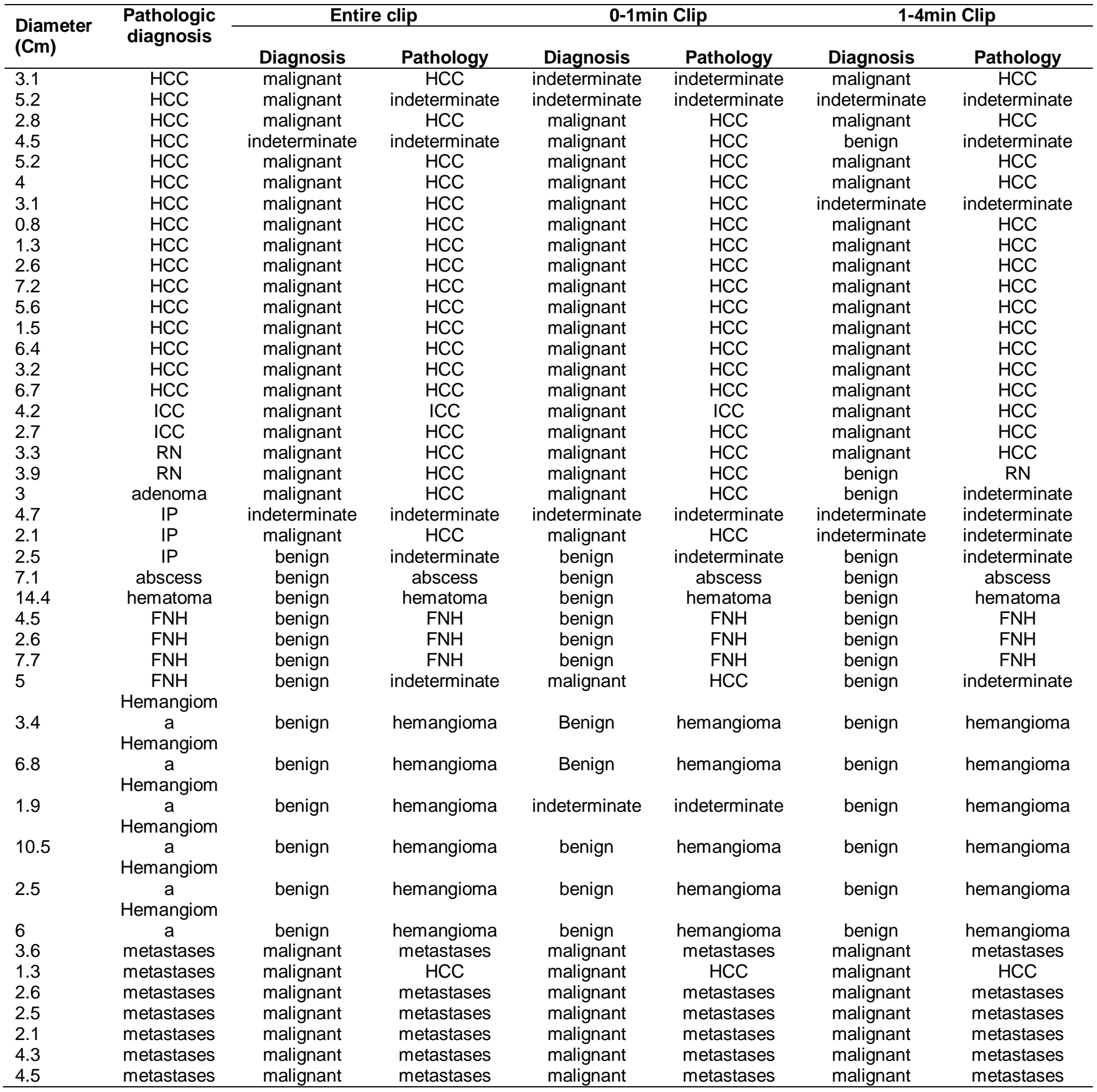

FLL, focal liver lesion; HCC, hepatocellular carcinomas; ICC, intrahepatic cholangiocarcinoma; IP, inflammatory pseudotumor; $\mathrm{RN}$, regenerative nodule; $\mathrm{FNH}$, focal nodular hyperplasias

Table 2: Results for entire clips, $0-1 \mathrm{~min}$ clips and 1-4min clips concerning benign or malignant diagnosis

\begin{tabular}{lccc}
\hline Variable & Correct diagnosis & \multicolumn{2}{c}{ Incorrect diagnosis } \\
\cline { 3 - 4 } & & Indeterminate & Misdiagnosis \\
\hline Entire clip & $38(88.4 \%)$ & $2(4.7 \%)$ & $3(7.0 \%)$ \\
$0-1$ min clip & $34(79.1 \%)$ & $4(9.3 \%)$ & $5(11.6 \%)$ \\
$1-4$ min clip & $37(86.1 \%)$ & $4(9.3 \%)$ & $2(4.7 \%)$ \\
\hline
\end{tabular}


Table 3: Results for entire clips, $0-1 \mathrm{~min}$ clips and 1-4min clips concerning pathological diagnosis

\begin{tabular}{lccc}
\hline & Correct diagnosis & \multicolumn{2}{c}{ Incorrect diagnosis } \\
\cline { 3 - 4 } Variable & & Indeterminate & Misdiagnosis \\
\hline entire clip & $32(74.4 \%)$ & $5(11.6 \%)$ & $6(14.0 \%)$ \\
0-1min clip & $31(72.1 \%)$ & $5(11.6 \%)$ & $7(16.3 \%)$ \\
$1-4$ min clip & $31(72.1 \%)$ & $8(18.6 \%)$ & $4(9.3 \%)$ \\
\hline
\end{tabular}

Table 4: Correlation of analysis of $0-1 \mathrm{~min}$ and $1-4 \mathrm{~min}$ clip compared with the entire clip ( $P$-value)

\begin{tabular}{lcc}
\hline Variable & Benign/Malignant diagnosis & Pathological diagnosis \\
\hline $0-1 \mathrm{~min}$ clip & 0.243 & 0.808 \\
1-4min clip & 0.747 & 0.808 \\
\hline
\end{tabular}

To simplify the process and reduce the CEUS time, $0-1 \mathrm{~min}$ clips and $1-4 \mathrm{~min}$ clips were divided from entire clips in this study. Subsequently, no significant differences was found between $0-1 \mathrm{~min}$ or $1-4 \mathrm{~min}$ clips versus the entire clips with respect to malignant or benign diagnosis and pathological diagnosis, which means that $0-1 \mathrm{~min}$ clip and 1-4min clip can be used independently to reach valid diagnosis in CEUS of FLLs. Additionally, our study also demonstrated that it's possibility of CEUS for testing two different FLLs based on $0-1 \mathrm{~min}$ and $1-4 \mathrm{~min}$ clip with only one injection of contrast agent.

Typical haemangiomas will show peripheral nodular enhancement (PNE) in arterial phase of CEUS, whereas untypical haemangiomas would not observe PNE (especially seen in small haemangiomas), resulting in misdiagnosis by 0 $1 \mathrm{~min}$ clips, but the gray-scale ultrasound of hyperecho in normal liver tissue would help to obtain correct diagnosis $[6,9,10]$. In our study, for the 6 haemangiomapatients, $0-1 \mathrm{~min}$ clips obtained correct pathologic diagnosis in 5 patients, and $1-4 \mathrm{~min}$ and the entire clips obtained correct pathologic diagnosis in all the 6 patients. The CEUS characteristic of typical $\mathrm{FNH}$ is centrifugal filling with central spoke wheelshaped contrast enhancement during the arterial phase $[6,9,10]$. Thus the absence of central spoke wheel may result in wrong diagnosis within FNH nodules, which might be misdiagnosed as malignant lesions because of rapidly filling in the artery phase. However, it did not affect the diagnosis as benign nodules by the entire clips and 1-4min clips in our investigation.

The HCC multistep carcinogenesis could lead to changes in intra-nodular blood supply, consisting in a progressive decrease of the portal supply associated to a decrease in normal arterial supply, subsequently overcome by increase of newly formed tumoral arterial supply [11-13]. Generally, regenerative nodules are nearly isoenhancing in all phases but the not negligible rate of HCCs showing this same vascular pattern (the so-called "hypovascular HCC" approximately
10-20\%) [14]. The differential diagnosis among regenerative nodule, hepatocellular adenoma and HCC is not always easy to be reached, which imposes a strict recall strategy for any newly detected lesion in cirrhosis [15]. Contrast features of metastases at CEUS are hyperenhancement during the arterial phase (or rimlike enhancement with non-enhancing central areas in hypo-vascular metastases) followed by marked hypo-enhancement during the late phase [16]. Metastases especially small FLL, without hypo-enhancement, present homogenous enhancement, may influence the diagnosis by the entire clip and $0-1 \mathrm{~min}$ clip. But the history and appearance of multiply FLLs can do some help. Intrahepatic cholangiocarcinoma (ICC) is a tough diagnostic challenge duo to its asymptomatic clinical features and atypical imaging characteristics $[17,18]$. Commonly, it is more difficult to distinguish smaller ICC (diameter $\leq 3 \mathrm{~cm}$ ) from HCC on CEUS, corresponding to more frequently appeared homogeneous hyperenhancement [19]. In our study, similar with the entire clips, the $0-1 \mathrm{~min}$ clips could correct diagnose 14 in 16 patients. In addition, for the 7 patients with liver metastases, all the 0-1min, 1-4 min and entire clips could correct diagnose 6 patients.

Inflammatory pseudotumor (IP) is an uncommon benign lesion with a variable degree of CEUS enhancement appearance [20]. In this study, one IP with absent contrast enhancement was diagnosed correctly, while two IPs with enhancement in arterial phase and hypoechoic appearance in late phases were misdiagnosed as HCC or indeterminate lesion. This phenomenon has been seen in another study [21]. The various CEUS patterns of IP were deemed to relate with different quantity of cellular areas and intense fibrosis areas [20,22]. Unfortunately, until now, there has been no systematic report on the CEUS appearance of IP, which needs further study. In routine clinical practice, a definitive and exact differential diagnosis of FLLs with CEUS entire clip is possible only in some types of lesions, whilst the 
simple categorization of a lesion as benign or malignant can be achieved with extremely high sensitivity (>90\%) and specificity [2], as well as with an accuracy comparable to CT or MRI in most series [23-26]. Additionally, in typical lesions the $0-1 \mathrm{~min}$ clip and $1-4 \mathrm{~min}$ clip could be comparable to the entire clip in diagnosis of FLLs aforementioned.

However, we could see that some lesions might have atypical contrast-enhancement patterns (ACEP), which may lead to misdiagnosis. Between 5 and $25 \%$ FLLs remains indeterminate even after CEUS (especially in cirrhotic livers) $[5,6,9,21,27,28]$. These situations could go against the $0-1 \mathrm{~min}$ clip and $1-4 \mathrm{~min}$ clip derived from the entire CEUS clip much more seriously. Thus, the ACEP FLLs need to be characterized by other diagnostic investigations.

\section{Limitations of the study}

First, the main limitation of the study was that $s$ other FLLs were not included in the study and some of them may be difficult to differentiate from each other $[29,30]$. Therefore, the results of the study are only applicable for FLLs mentioned above. Second, since the FLLs number in our study is limited, the usefulness of $0-1 \mathrm{~min}$ clip and 1-4min clip in diagnosing FLLs is needed further investigation with large cases. Third, these findings might be related to the fact that both readers were blinded to the liver etiology history, elevation of serum biomarkers such as alphafetoprotein or CA, and other laboratory findings, as well as other imaging findings or clinical presentations. The authors believe that precise patient history and/or further examinations are mandatory to reach a correct differential diagnosis. In addition, the experiences of radiologists in CEUS will influence the diagnosis of FLL.

\section{CONCLUSION}

The findings of this study demonstrate that there is no significant difference between CEUS entire clip, 0-1min clip and 1-4 min clip in identifying FLLs, and based on which the diagnosis of two different FLLs during CEUS with only one injection of contrast agent can be available. The findings also demonstrate that the appearance of lesions in the arterial phase is also very important when distinguishing between benign and malignant lesions, although appearance in the portal and late phases is important when characterizing them.

\section{REFERENCES}

1. Wilson SR, Greenbaum LD, Goldberg BB. Contrastenhanced ultrasound: what is the evidence and what are the obstacles? AJR Am J Roentgenol 2009; 193(1): 5560.

2. Quaia E. The real capabilities of contrast-enhanced ultrasound in the characterization of solid focal liver lesions. Eur Radiol 2011; 21(3): 457-462.

3. Claudon M, Dietrich CF, Choi BI. Guidelines and good clinical practice recommendations for Contrast Enhanced Ultrasound (CEUS) in the liver - update 2012: A WFUMB-EFSUMB initiative in cooperation with representatives of AFSUMB, AIUM, ASUM, FLAUS and ICUS. Ultrasound Med Biol 2013; 39(2): 187-210.

4. Jang JY, Kim MY, Jeong SW, Kim TY, Kim SU, Lee SH, Suk KT, Park SY, Woo HY, Kim SG, Heo J. Current consensus and guidelines of contrast enhanced ultrasound for the characterization of focal liver lesions. Clin Mol Hepatol 2013; 19(1): 1-16.

5. Bryant TH, Blomley MJ, Albrecht T, Sidhu PS, Leen EL, Basilico R, Pilcher JM, Bushby LH, Hoffmann CW. Improved characterization of liver lesions with liverphase uptake of liver-specific microbubbles: prospective multicenter study. Radiology 2004; 232(3): 799-809.

6. Quaia E, Calliada F, Bertolotto M, Rossi S, Garioni L, Rosa L, Pozzi-Mucelli R. Characterization of focal liver lesions with contrast-specific US modes and a sulfur hexafluoride-filled microbubble contrast agent: diagnostic performance and confidence. Radiology 2004; 232(2): 420-430.

7. Goertz RS, Bernatik T, Strobel D, Hahn EG, Haendl $T$. Software-based quantification of contrast-enhanced ultrasound in focal liver lesions--a feasibility study. Eur J Radiol 2010; 75(2): e22-26.

8. Huang-Wei C, Bleuzen A, Bourlier P, Roumy J, Bouakaz $A$, Pourcelot L, Tranquart F. Differential diagnosis of focal nodular hyperplasia with quantitative parametric analysis in contrast-enhanced sonography. Invest Radiol2006; 41(3): 363-368.

9. Dai Y, Chen MH, Yin SS, Yan K, Fan ZH, Wu W, Wang $Y B$, Yang W. Focal liver lesions: Can SonoVueenhanced ultrasound be used to differentiate malignant from benign lesions? Invest Radiol 2007; 42(8): 596603.

10. Kim TK, Jang HJ, Burns PN, Murphy-Lavallee J, Wilson SR. Focal nodular hyperplasia and hepatic adenoma: differentiation with low-mechanical-index contrastenhanced sonography. AJR Am J Roentgenol2008; 190(1):58-66.

11. Leoni S, Piscaglia F, Golfieri R, Camaggi V, Vidili G, Pini $P$, Bolondi $L$. The impact of vascular and nonvascular findings on the noninvasive diagnosis of small hepatocellular carcinoma based on the EASL and AASLD criteria. Am J Gastroenterol2010; 105(3):599609. 
12. Kudo M. Multistep human hepatocarcinogenesis: correlation of imaging with pathology. J Gastroenterol2009; 44 Suppl 19:112-118.

13. Kumada T, Nakano S, Toyoda H, Hayashi K, Kiriyama S, Sone $Y$, Tanikawa $M$, Hisanaga $Y$, Yamamoto $A$, Takeshima K. Assessment of tumor hemodynamics in small hepatocellular carcinoma: comparison of Doppler ultrasonography, angiography-assisted computed tomography, and pathological findings. Liver Int2004; 24(5): 425-431.

14. Bolondi L, Gaiani S, Celli N, Golfieri R, Grigioni WF, Leoni S, Venturi AM, Piscaglia F. Characterization of small nodules in cirrhosis by assessment of vascularity: the problem of hypovascular hepatocellular carcinoma. Hepatology 2005; 42(1): 27-34.

15. Piscaglia F, Lencioni R, Sagrini E. Characterization of focal liver lesions with contrast-enhanced ultrasound. Ultrasound Med Biol 2010; 36(4): 531-550.

16. Murphy-Lavallee J, Jang HJ, Kim TK, Burns PN, Wilson $S R$. Are metastases really hypovascular in the arterial phase? The perspective based on contrast-enhanced ultrasonography. J Ultrasound Med 2007; 26(11): 15451556.

17. Chen $L D, X u H X$, Xie $X Y$, Xie $X H, X u Z F$, Liu GJ, Wang $Z$, Lin MX, Lu MD. Intrahepatic cholangiocarcinoma and hepatocellular carcinoma: differential diagnosis with contrast-enhanced ultrasound. Eur Radiol 2010; 20(3):743-753.

18. $X u H X$, Chen $L D$, Xie $X Y$, Xie $X H, X u Z F$, Liu GJ, Lin $M X$, Wang $Z, \quad L u$ MD. Enhancement pattern of hilarcholangiocarcinoma: contrast-enhanced ultrasound versus contrast-enhanced computed tomography. Eur J Radiol 2010; 75(2): 197-202.

19. Vilana R, Forner A, Bianchi L. Intrahepatic peripheral cholangiocarcinoma in cirrhosis patients may display a vascular pattern similar to hepatocellular carcinoma on contrast-enhanced ultrasound. Hepatology 2010; 51(6):2020-2029.

20. Chen Y, Jiang TA, Ao JY. Contrast-enhanced ultrasonography in diagnosis of inflammatory pseudotumor of liver. Zhejiang Da Xue Xue Bao 2010; 39(6): 634-637.

21. Blomley MJ, Sidhu PS, Cosgrove DO. Do different types of liver lesions differ in their uptake of the microbubble contrast agent SH U 508A in the late liver phase? Early experience. Radiology 2001; 220(3): 661-667.
22. Nam KJ, Kang HK, and Lim JH. Inflammatory pseudotumor of the liver: $C T$ and sonographic findings. AJR Am J Roentgenol 1996; 167(2): 485-487.

23. Trillaud H, Bruel JM, Valette PJ. Characterization of focal liver lesions with SonoVue-enhanced sonography: international multicenter-study in comparison to CT and MRI. World J Gastroenterol 2009; 15(30): 3748-3756.

24. Seitz K, Strobel D, Bernatik T, Blank W, Friedrich-Rust M, Herbay Av, Dietrich CF, Strunk H, Kratzer W, Schuler A. Contrast-Enhanced Ultrasound (CEUS) for the characterization of focal liver lesions - prospective comparison in clinical practice: CEUS vs. CT (DEGUM multicenter trial). Parts of this manuscript were presented at the Ultrasound Dreilandertreffen 2008, Davos. Ultraschall Med 2009; 30(4):383-389.

25. Seitz K, Bernatik T, Strobel D, Blank W, Friedrich-Rust M, Strunk $H$, Greis C, Kratzer W, Schuler A. Contrastenhanced ultrasound (CEUS) for the characterization of focal liver lesions in clinical practice (DEGUM Multicenter Trial): CEUS vs. MRI--a prospective comparison in 269 patients. Ultraschall Med 2010; 31(5): 492-499.

26. Strobel D, Seitz K, Blank W, Schuler A, Dietrich $C$, von Herbay A, Friedrich-Rust $M$, Kunze G. Contrastenhanced ultrasound for the characterization of focal liver lesions--diagnostic accuracy in clinical practice (DEGUM multicenter trial).Ultraschall Med 2008; 29(5): 499-505.

27. von Herbay A, Vogt $C$, Haussinger D. Late-phase pulseinversion sonography using the contrast agent levovist: differentiation between benign and malignant focal lesions of the liver. AJR Am J Roentgenol 2002; 179(5): 1273-1279.

28. von Herbay A, Westendorff J, and Gregor M. Contrastenhanced ultrasound with SonoVue: differentiation between benign and malignant focal liver lesions in 317 patients. J Clin Ultrasound2010; 38(1): 1-9.

29. Foschi FG, Dall'Aglio AC, Marano G, Lanzi A, Savini $P$, Piscaglia F, Serra C, Cursaro C, Bernardi M, Andreone $P$, Stefanini GF. Role of contrast-enhanced ultrasonography in primary hepatic lymphoma. J Ultrasound Med 2010; 29(9):1353-1356.

30. Luo XL, Liu D, Yang JJ, Zheng MW, Zhang J, Zhou XD. Primary gastrointestinal stromal tumor of the liver: a case report. World J Gastroenterol2009; 15(29):37043707. 